\title{
Applications of Plasmon Energy Expansion Thermometry
}

\author{
Matthew Mecklenburg ${ }^{1}$, William A. Hubbard ${ }^{2}$, E. R. White ${ }^{2}$, Rohan Dhall ${ }^{3}$, Stephen B. Cronin ${ }^{3}$, Shaul \\ Aloni $^{4}$, and B. C. Regan ${ }^{2}$ \\ 1. Center for Electron Microscopy and Microanalysis, University of Southern California, Los Angeles, \\ CA, USA. \\ 2. Department of Physics and Astronomy \& California NanoSystems Institute, University of California, \\ Los Angeles, CA, USA. \\ 3. Department of Electrical Engineering, University of Southern California, Los Angeles, CA, USA \\ 4. Molecular Foundry, Lawrence Berkeley National Laboratory, Berkeley, CA, USA.
}

Plasmon energy expansion thermometry (PEET) has finer spatial resolution than conventional thermometers such as thermocouples, optical pyrometers, and resistance thermometers [1]. In an electron microscope, a beam electron interacting with a sample sometimes loses energy by creating a plasmon, or charge oscillation. A plasmon's energy is proportional to the square root of the sample material's valence electron density. Thermal expansion changes this density and thereby shifts the plasmon energy [2]. PEET relates the plasmon energy, which can be mapped with few-nanometer spatial resolution using electron energy loss spectroscopy (EELS), to temperature. Such high-spatial resolution thermometry has numerous applications in electron microscopy [3], including heating holder calibration and temperature gradient mapping.

In a heating sample holder, whether it is furnace-style or has a microfabricated heater membrane, the temperatures are not always uniform and well-characterized. Both in situ and external temperature calibrations have associated uncertainties. In the former case the thermometer cannot be co-located with the sample (it is often quite remote), and in the latter the calibration may change.

PEET can calibrate sample temperature as a function of heater power directly (Fig. 1). Examples of plasmon energy maps acquired for PEET are shown in Figs. 1E-F, along with the simultaneously acquired annular dark field (ADF) images (Figs. 1C-D). Both Fig. 1E and Fig. 1F show a network of grain boundaries, which give $10-20 \mathrm{meV}$ plasmon energy shifts due to the local density decrease. (The detection of these grain boundaries demonstrates that PEET is sensitive to density changes that occur on atomic length scales.) Figure $1 \mathrm{E}$ is the reference (room temperature) map. During the acquisition of the Fig. 1F map, the heater power was stepped (Fig. 1A), which produced the plasmon shifts appearing as dark bands. The small heater induces little thermal drift. Combining the data of Figs. 1E-F [1] gives the temperature change (Fig. 1G). The heater power vs temperature plot (Fig. 1H) represents a local, in situ temperature calibration. Here a continuous aluminum film was used, but nanoparticles of aluminum or another material with a well-defined plasmon could be dispersed on a sample to provide point thermometry within the field of view.

PEET can also map the temperature gradients in an operating microelectronic device such as the aluminium heater shown in Fig. 2. With increasing power dissipation the temperature gradients in the aluminium wire become steeper. The wire is hottest in the center, much like an incandescent bulb's filament. PEET adds a new temperature mapping capability, on par with structure determination and elemental analysis, to the already extensive capabilities of TEM for characterizing microelectronic devices [4]. 
References:

[1] M Mecklenburg et al, Science 347 (2015), p. 629-632.

[2] G Meyer, Zeitschrift fur Physik 148 (1957), p. 61-71.

[3] M P Seah et al, Journal of Materials Science 21 (1986), p. 1305-1309.

[4] This work was supported by NSF DMR-1206849, and in part by FAME, one of six centers of STARnet, a Semiconductor Research Corporation program sponsored by MARCO and DARPA. Data presented were acquired at the Center for Electron Microscopy and Microanalysis at the University of Southern California.
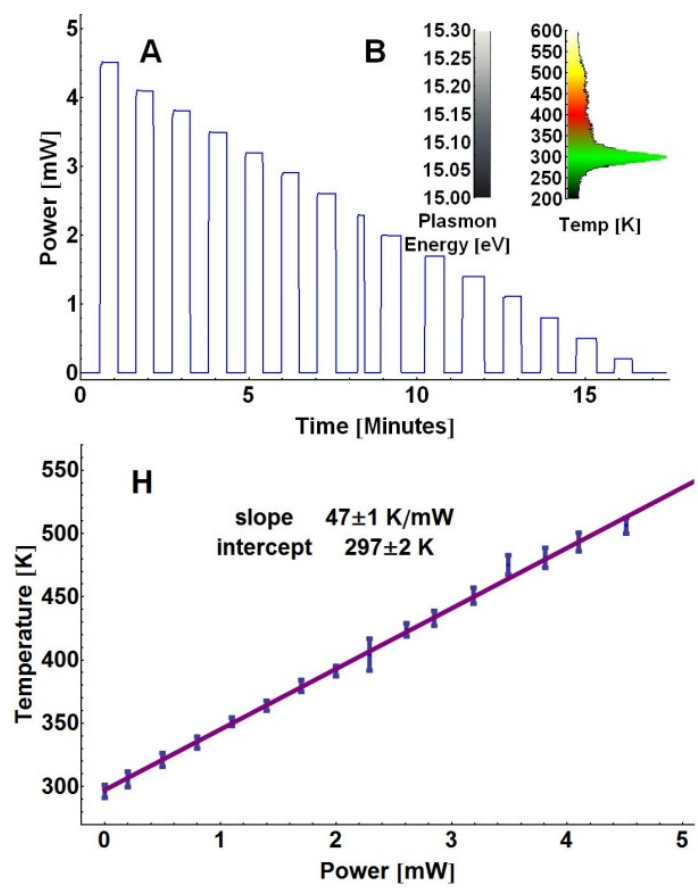
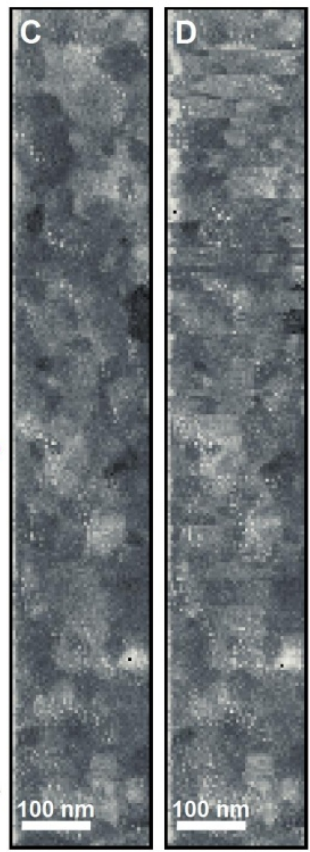
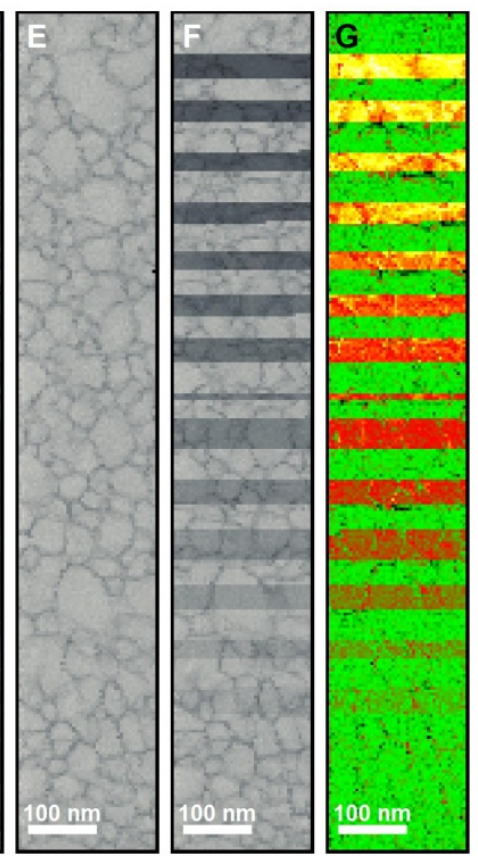

Figure 1. (A) A plot of power vs time during the EELS scan. (B) An intensity scale for the plasmon maps (E) and (F) and temperature map (G). Images (C) and (D) show the ADF images simultaneously acquired with the plasmon maps in $(\mathrm{E})$ and $(\mathrm{F})$. The temperature map resulting from $(\mathrm{E})$ and $(\mathrm{F})$ is shown in $(\mathrm{G})$. Each map is $46 \times 271$ pixels with a $5 \mathrm{~nm}$ pixel pitch.
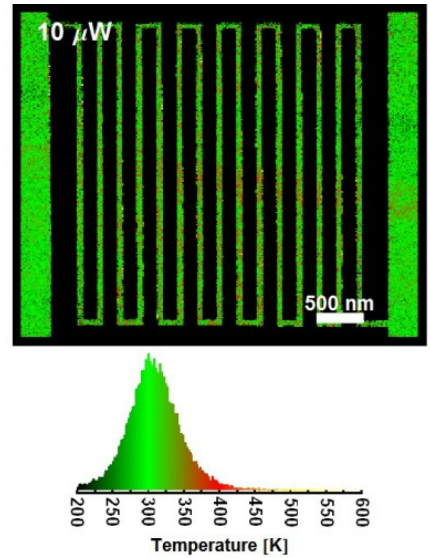
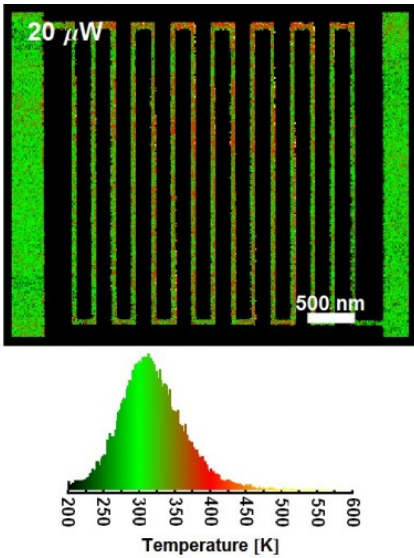
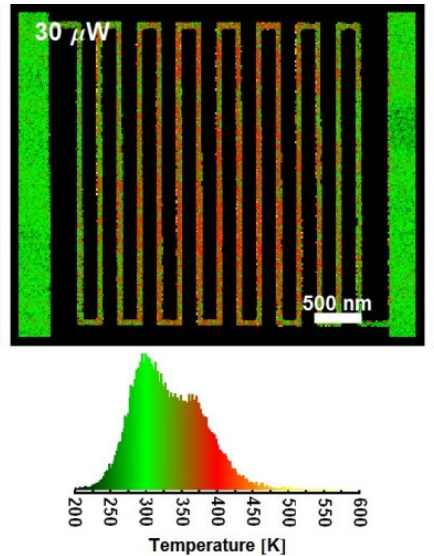
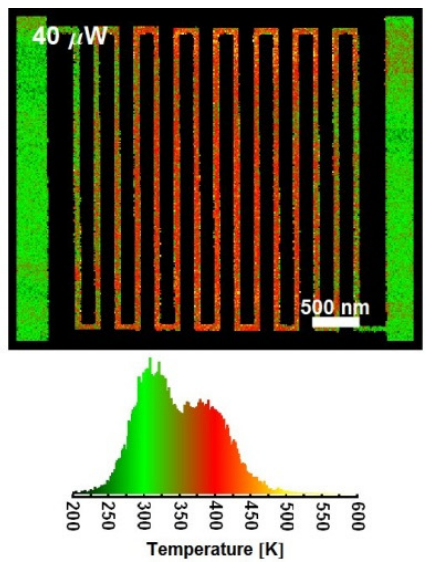

Figure 2. Four maps at different powers are shown. Each map is $313 \times 256$ pixels in size with a $17 \mathrm{~nm}$ pixel pitch. The histogram of temperatures from each image is shown below the corresponding image. 